\title{
Gastrokine 1 protein is a potential theragnostic target for gastric cancer
}

\author{
Jung Hwan Yoon ${ }^{1,2} \cdot$ In-Hye Ham ${ }^{3,4}$. Olga Kim ${ }^{1} \cdot$ Hassan Ashktorab ${ }^{5}$. Duane T. Smoot ${ }^{6} \cdot$ Suk Woo Nam $^{1,2}$. \\ Jung Young Lee ${ }^{1,2} \cdot$ Hoon Hur ${ }^{3,4} \cdot$ Won Sang Park ${ }^{1,2}$
}

Received: 29 December 2017 / Accepted: 19 April 2018 / Published online: 27 April 2018

(c) The Author(s) 2018

\begin{abstract}
Background Gastrokine 1 (GKN1) plays important roles in maintaining mucosal homeostasis, and in regulating cell proliferation and differentiation. Here, we determined whether GKN1 is a potential theragnostic marker for gastric cancer.

Methods We identified GKN1 binding proteins using the protein microarray assay and investigated whether GKN1 is one of the exosomal cargo proteins by western blot, immunoprecipitation, and immunofluorescent assays. Cell proliferation and apoptosis were analyzed by MTT, BrdU incorporation, flow cytometry, and western blot assays. We further validated the functional relevance of exosomal GKN1 in MKN1-injected xenograft mice. The possibility of serum GKN1 as a diagnostic marker for gastric cancer was determined by ELISA assay.

Results In protein microarray assay, GKN1 binding to 27 exosomal proteins was clearly observed. GKN1 was expressed in exosomes derived from HFE-145 gastric epithelial cells by western blot and immunofluorescent assays, but not in exosomes from AGS and MKN1 gastric cancer cells. Exosomes carrying GKN1 inhibited cell proliferation and induced apoptosis in both AGS and MKN1 cells, and exosomes carrying GKN1-treated nude mice-bearing MKN1 xenograft tumors exhibited significantly reduced tumor volume and tumor weight. Silencing of clathrin markedly down-regulated the internalization of exosomal GKN1. Interestingly, serum GKN1 concentrations in patients with gastric cancer were significantly lower than those in healthy individuals and patients with colorectal and hepatocellular carcinomas.

Conclusions The GKN1 is secreted and internalized in the gastric epithelium by exosome-driven transfer, which inhibits gastric tumorigenesis and supports the clinical application of GKN1 protein in gastric cancer diagnosis and treatment.
\end{abstract}

Keywords GKN1 $\cdot$ Exosome $\cdot$ Theragnosis $\cdot$ Gastric cancer

Electronic supplementary material The online version of this article (https://doi.org/10.1007/s10120-018-0828-8) contains supplementary material, which is available to authorized users.

Hoon Hur

hhcmc75@ajou.ac.kr

$\triangle$ Won Sang Park

wonsang@catholic.ac.kr

1 Department of Pathology, College of Medicine, The Catholic University of Korea, 222 Banpo-daero, Seocho-gu, Seoul 06591, South Korea

2 Functional RNomics Research Center, College of Medicine, The Catholic University of Korea, 222 Banpo-daero, Seocho-gu, Seoul 06591, South Korea

\section{Introduction}

Human gastrokine 1 (GKN1), made of 185 amino acid residues, is a stomach-specific protein which is produced by gastric mucus-secreting cells, stored in cytoplasmic granules, and secreted with mucus onto the apical cell surface

3 Department of Surgery, Ajou University School of Medicine, Suwon 16499, South Korea

4 Brain Korea 21 Plus Research Center for Biomedical Science, Ajou University, Suwon 16499, South Korea

5 Department of Medicine, Howard University, Washington, DC 20060, USA

6 Department of Medicine, Meharry Medical Center, Nashville, TN 37208, USA 
$[1,2]$. We and others previously showed that inactivation of GKN1 as a tumor suppressor is one of the leading causes of gastric cancer [3-5]. Although GKN1 plays important roles in maintaining mucosal integrity and homeostasis, and in regulating cell proliferation and differentiation [6-8], no study has yet investigated how GKN1 secretion and internalization take place in gastric mucosal epithelium.

Recently, it has been reported that intercellular communication through the transfer of extracellular vesicles (EVs) plays an important role in a range of physiological processes including intercellular exchange of proteins and RNA [9, $10]$ and immune regulation $[10,11]$ in multicellular organisms. EVs are small vesicles released by donor cells that can be taken up by recipient cells [12]. EVs that are derived from multi-vesicular bodies are referred to as exosomes [13]. Exosomes can carry cargo from their originating cells as functional messenger RNA, microRNA, or proteins [14, 15]. Many EV proteins have been shown to interact with membrane receptors on target cells $[16,17]$ and are usually taken up into endosomal compartments via endocytosis [18-20]. To understand the emerging roles of exosomes in normal physiological processes and in diseases, it is important to identify the molecular mechanisms that underlie exosome release by donor cells and exosome internalization by target cells.

Here, we provided the first evidence that human gastric epithelial cells naturally secrete and internalize GKN1 as an exosomal protein and that GKN1 protein may be a potential diagnostic marker and therapeutic target for gastric cancer.

\section{Methods}

\section{Cell culture and transfection of c-Myc}

Cell culture and transfection of c-Myc were described in the supplementary Methods.

\section{Identifying the GKN1-binding proteins through HuProtTM microarrays}

Identifying the GKN1-binding proteins through HuProtTM microarrays were described in the supplementary Methods.

\section{Co-immunoprecipitation}

We washed exosomes derived from AGS and MKN1 cells treated with recombinant GKN1 protein ( $\mathrm{rGKN} 1)$ with PBS and lysed at $4{ }^{\circ} \mathrm{C}$ with PBS, pH 7.2 containing $1 \%$ NP-40, $0.5 \%$ sodium deoxycholate, $0.1 \% \mathrm{SDS}, 10 \mathrm{mMNaF}, 1.0 \mathrm{mM}$ $\mathrm{NaVO} 4$, and $1 \%$ protease inhibitor cocktail (Sigma, St. Louis, MO, USA), as described previously [21]. Equal protein aliquots $(1.0 \mathrm{mg})$ were immunoprecipitated with $2.0 \mu \mathrm{g}$ of antibodies to GKN1 (Abcam, Cambridge, UK), COMT and YWHAZ (Genetex, CA, USA) plus protein A/G-agarose (Santa Cruz Biotechnology), as described previously [22].

\section{Exosome isolation}

Exosome isolation and labeling were described in the supplementary Methods.

\section{Trypsin digestion assay}

To determine the localization of GKN1 in exosomes, we subjected purified exosomes from HFE-145 cells ( $35 \mu \mathrm{g}$ proteins in $7 \mu \mathrm{L}$ of PBS) to lipid membrane lysis by $1 \%$ Triton $\mathrm{X}-100$ for $30 \mathrm{~min}$ at $4{ }^{\circ} \mathrm{C}$, as previously described [23]. A detailed description for trypsin digestion assay is in the supplementary Methods.

\section{Immunoblot, immunofluorescent, and transmission electron microscopy (TEM) studies}

Immunoblot, immunofluorescent, and transmission electron microscopy assays were described in the supplementary Methods.

\section{Real-time RT-PCR}

Real-time RT-PCR analysis was described in the supplementary Methods.

\section{Measurement of cell viability and apoptosis}

Measurement of cell viability and apoptosis were described in the supplementary Methods.

\section{Flow-cytometry analysis of cell cycle}

Flow-cytometry analysis of cell cycle was described in the supplementary Methods.

\section{Chromatin immunoprecipitation (ChIP)}

To assess the c-Myc and NKX6.3 binding in the promoter regions of NKX6.3 and GKN1, respectively, we performed ChIP assays using the Thermo Scientific Pierce Agarose ChIP kit (Thermo Scientific Pierce), as described previously [22]. We amplified the DNA by PCR using primers for the NKX6.3 promoter, as described in supplementary Table $\mathrm{S} 1$. We separated amplification products on a $2 \%$ agarose gel. 


\section{In vivo assay}

Animal experiments were approved and performed in accordance with the Institutional Animal Care and Use Committee at the Ajou University School of Medicine (Suwon, Gyeonggi-do, Korea). A detailed description for in vivo assay was in the supplementary Methods.

\section{Serum samples}

We obtained written informed consent from all participants in accordance with the Declaration of Helsinki. The study was approved by the Institutional Review Board of The Catholic University of Korea, College of Medicine (MC16SISI0132). There was no evidence of familial cancer in any of the patients. A detailed description for serum samples was in the supplementary Methods.

\section{Statistical analysis}

We used Student's $t$ test to analyze the effects of GKN1 on cell viability and proliferation, and Chi-square test to compare GKN1, Ki-67, and Annexin V staining patterns. We performed all experiments in duplicate to verify the reproducibility of the findings. Data are expressed as means $\pm \mathrm{SD}$ from two independent experiments. We considered a $P$ value less than 0.05 to be the limit of statistical significance. To further evaluate the diagnostic usefulness of the markers based on dichotomous classification, we conducted receiver operating characteristic (ROC) curve analysis. An ROC curve is a plot of the true-positive fraction versus the falsepositive fraction evaluated for all possible cut-off point.

\section{Results}

\section{Identification of GKN1-binding proteins}

In protein microarray assay, we clearly observed that recombinant GKN1 (rGKN1) bound to 27 exosomal proteins; the GKN1-interacting exosomal proteins are presented in Fig. 1a. Of these, we verified the interaction between GKN1 and COMT and between GKN1 and YWHAZ using coimmunoprecipitation analysis (Fig. 1b). a

\begin{tabular}{|c|c|c|c|c|c|}
\hline Number & Gene symbol & Description & Entrez ID & Chromosome & Map location \\
\hline 1 & ACSL4 & acyl-CoA synthetase long-chain family member 4 & 2182 & $\mathrm{x}$ & $\mathrm{Xq22.3-q23}$ \\
\hline 2 & AHNAK & AHNAK nucleoprotein & 79026 & 11 & 11912.2 \\
\hline 3 & ALDHIL1 & aldehyde dehydrogenase 1 family, member L1 & 10840 & 3 & 3921.3 \\
\hline 4 & ALDH9A1 & aldehyde dehydrogenase 9 family, member A1 & 223 & 1 & 1923.1 \\
\hline 5 & ASNA1 & arsA arsenite transporter, ATP-binding, homolog 1 (bacterial) & 439 & 19 & $19 q 13.3$ \\
\hline 6 & СКB & creatine kinase, brain & 1152 & 14 & 14q32 \\
\hline 7 & CLC1 & chloride intracellular channel 1 & 1192 & 6 & 6p21.3 \\
\hline 8 & сомт & catechol-o-methytransferase & 1312 & 22 & 22917.21 \\
\hline g & CTNS & cystinosin, Iysosomal cystine transporter & 1497 & 17 & $17 \mathrm{p} 13$ \\
\hline 10 & GGPD & glucose-6-phosphate dehydrogenase & 2539 & $\mathrm{x}$ & Xa28 \\
\hline 11 & GALM & galactose mutarotase (aldose 1-epimerase) & 130589 & 2 & $2 p 22.1$ \\
\hline 12 & GARS & glycyl-tRNA synthetase & 2617 & 7 & $7 p 15$ \\
\hline 13 & GLUL & glutamate-ammonia ligase & 2752 & 1 & 1931 \\
\hline 14 & GPD1 & glycerol-3-phosphate dehydrogenase 1 (soluble) & 2819 & 12 & $12 q 13.12$ \\
\hline 15 & GSN & gelsolin & 2934 & 9 & 9933 \\
\hline 16 & GSTM3 & glutathione S-transferase mu 3 (brain) & 2947 & 1 & 1p13.3 \\
\hline 17 & HRAS & Harvey rat sarcoma viral oncogene homolog & 3265 & 11 & $11 p 15.5$ \\
\hline 18 & IL1RN & interleukin 1 receptor antagonist & 3557 & 2 & 2914.2 \\
\hline 19 & PGD & phosphogluconate dehydrogenase & 5226 & 1 & $1 p 36.22$ \\
\hline 20 & PKLR & pyrruvate kinase, liver and $R B C$ & 5313 & 1 & 1921 \\
\hline 21 & PSAT1 & phosphoserine aminotransferase 1 & 29968 & 9 & $9 \mathrm{q} 21.2$ \\
\hline 22 & PSMBG & proteasome (prosome, macropain) subunit, beta type, 6 & 5694 & 17 & 17p13 \\
\hline 23 & RNH1 & ribonuclease/angiogenin inhibitor 1 & 6050 & 11 & $11 \mathrm{p} 15.5$ \\
\hline 24 & SEC13 & SEC13 homolog (S. cerevisiae) & 6396 & 3 & 3p25-p24 \\
\hline 25 & SPRR3 & small proline-rich protein 3 & 6707 & 1 & $1,921-q 22$ \\
\hline 26 & TGM1 & transglutaminase 1 & 7051 & 14 & $14 q 11.2$ \\
\hline 27 & YWHAZ & tyrosine 3-monooxygenase/tryptophan 5-monooxygenase activation protein, zeta & 7534 & 8 & 8923.1 \\
\hline
\end{tabular}

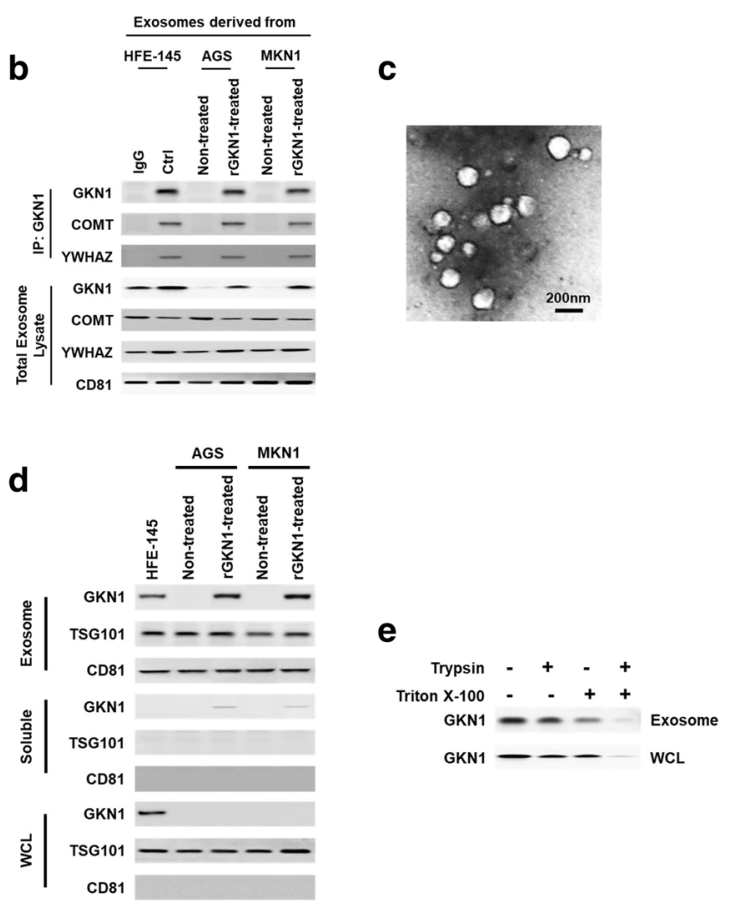

Fig. 1 Identification of GKN1-binding proteins and localization of GKN1 in exosomes. a In protein microarray, rGKN1 interacted with 27 exosomal proteins. b Exosomal GKN1 bound to COMT and YWHAZ. Immunoprecipitated proteins were resolved by sodium dodecyl sulfate-polyacrylamide gel electrophoresis, transferred to polyvinylidene difluoride membranes, and processed for immunoblotting with the indicated antibodies. c TEM image of exosomes isolated from supernatants of HFE-145 cells. d Representative western blots of GKN1, TSG-101, and CD81 in exosomes isolated from the
HFE-145-immortalized gastric epithelial cells, AGS and MKN1 gastric cancer cells. We found no GKN1 expression in exosomes derived from AGS and MKN1 cells, but we detected GKN1 expression in exosomes derived from HFE-145 cells as well as AGS and MKN1 cells treated with rGKN1. Soluble; exosome-free media, WCL; exosome-free whole cell lysate. e In enzymatic degradation assays, we treated exosomes derived from HFE-145 cells with trypsin and/or Triton X-100. When we treated them with both trypsin and Triton X-100, GKN1 protein was completely degraded 


\section{Detection of GKN1 in exosomes derived from gastric cell lines}

To investigate whether GKN1 is spontaneously secreted and internalized as an exosomal protein, we isolated exosomes from the cultured supernatants from HFE-145, AGS, and MKN1 gastric cells. In transmission electron microscopy (TEM) analysis, structurally intact exosomes, which revealed the presence of vesicles ranged in size from 30 to $200 \mathrm{~nm}$, could be detected (Fig. 1c). Expectedly, exosomes derived from HFE-145 immortalized gastric epithelial cells expressed GKN1, whereas exosomes from AGS and MKN1 gastric cancer cells did not (Fig. 1d). When we treated AGS and MKN1 cells with rGKN1, the expression of GKN1 was present mainly in the exosomes and scant or absent in exosome-free media and exosome-free whole cell lysate (Fig. 1d). Of two exosomal protein markers, we detected TSG101 in both the purified exosomes and exosome-free whole cell lysates, whereas we found CD81 expression only in exosomes (Fig. 1d).

Next, to reveal whether or not GKN1 protein is present in exosomes, we performed trypsin digestion assays with and without Triton X-100, followed by western blot analysis [23]. When we treated the exosomes with trypsin in the absence of Triton X-100, GKN1 was protected from trypsin-dependent enzymatic degradation. However, GKN1 was completely degraded in the presence of both trypsin and Triton X-100 (Fig. 1e), suggesting that GKN1 protein is present in exosomes.

\section{Measurement of cell proliferation and cell death}

To identify the biological effects of exosomal GKN1 on cell viability and proliferation, we treated HFE-145, AGS and MKN1 cells with exosomes derived (1) from AGS and MKN1 cells non-treated and treated with rGKN1, and (2) from HFE- 145 cells that originally expressed GKN1. Treatment with GKN1-negative exosomes derived from AGS and MKN1 cancer cells markedly increased cell viability in HFE-145, AGS and MKN1 cells, whereas GKN1-positive exosomes derived from same cells significantly inhibited cell viability and proliferation in AGS and MKN1 cells (Fig. 2a, b and supplementary Fig. 1a, b). Compared with rGKN1, exosomal GKN1 dramatically inhibited cell viability and proliferation in AGS and MKN1 cancer cells (Fig. 2c and supplementary Fig. 1c, d). In cell cycle analysis, exosomes derived from HFE-145 cells significantly decreased S phase and increased G1 and G2/M phase cell populations (Fig. 2d). Consistently, GKN1-positive exosomes derived from AGS cells treated with rGKN1 inhibited cell cycle progression in AGS and MKN1 cells (supplementary Fig. 2a). For G1 phase arrest, exosomal GKN1 increased expression of negative regulators of cell cycle such as p53 and p21 and down-regulated expression of positive regulators of cell cycle such as cdk4 and cyclin D (Fig. 2e). For G2/M arrest, GKN1 down-regulated expression of p-cdc2, cdc25c, and cyclin B proteins in HFE-145, AGS, and MKN1 cells (Fig. 2e).

In addition, GKN1-positive exosomes derived from HFE145 cells and AGS cells treated with rGKN1 significantly increased apoptotic AGS and MKN1 cells (Fig. 2f and supplementary Fig. 2b). Expression of cleaved caspase-3 and -8 increased markedly, whereas there were no significant changes in BAX or BCL2 expression (Fig. 2e). Taken together, it is likely that upon cellular internalization, exosomal GKN1 interacts with cytoplasmic proteins that inhibit proliferation and induce apoptosis of gastric epithelial cells.

To further confirm that exosomal GKN1 inhibits cell proliferation, we performed MTT and BrdU incorporation assays after treatment with GW4869 (sigma), an inhibitor of exosome biogenesis/release. In MKN1 cells, GW4869 markedly decreased exosomal protein concentration (Fig. 2g). As expected, rGKN1 significantly decreased cell viability and proliferation in MKN1 cells, whereas treatment with both rGKN1 and GW4869 did not (Fig. 2h and supplementary Fig. 1e, f). Thus, exosomal form of GKN1 protein may function as a tumor suppressor and serve as a therapeutic target of gastric cancer.

\section{GKN1 positive exosomes suppress tumor growth in vivo}

To investigate the effect of the tumor suppressive function of GKN1-positive exosomes in vivo, we intravenously injected the exosomes with or without GKN1 into the mice with MKN1 xenograft tumors subcutaneously six times in 2 weeks ( $n=7$ mice/group). The serum GKN1 concentration in GKN1-positive exosome-treated group was significantly higher relative to other groups (Fig. 3a). As expected, treatment with GKN1-positive exosomes significantly inhibited tumor growth by day 25 as compared to PBS- and control exosome-treated mice (Fig. 3b). Consistently, mean weight of harvested tumors (Fig. 3c) treated with GKN1-positive exosomes was significantly lower than that of tumors treated with control exosomes $(P=0.017)$ and PBS $(P=0.026)$ (Fig. 3d). Animal activity and change of body weight were not different among each treatment group during 4 week observation period (data not shown).

In immunofluorescent analysis, tumors from mice treated with GKN1-positive exosomes showed significantly increased GKN1 expression in the cytoplasm of cancer cells, but PBS- and control exosome-treated tumors did not (Fig. 3e). In addition, growth fraction with Ki-67 staining was significantly decreased in tumors treated with GKN1-positive exosomes $(P=0.018)$, whereas apoptosis with Annexin V staining was significantly higher compared 
a

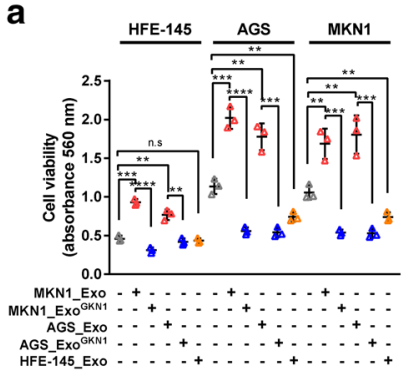

d
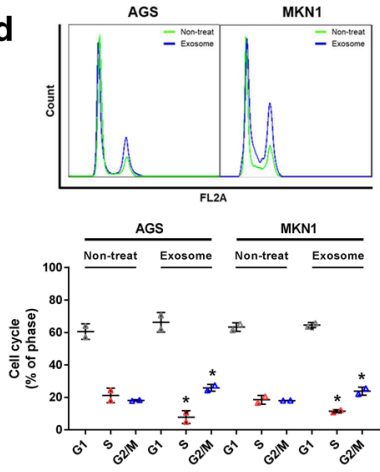

b

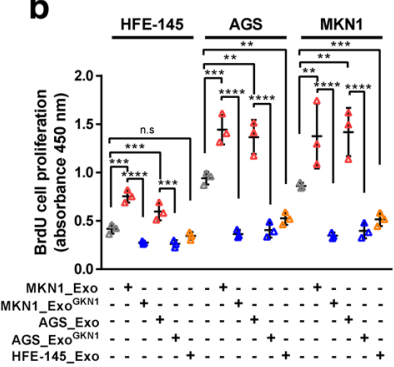

C

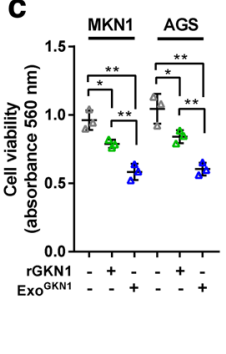

g

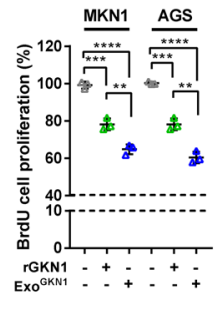

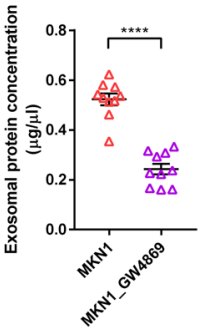

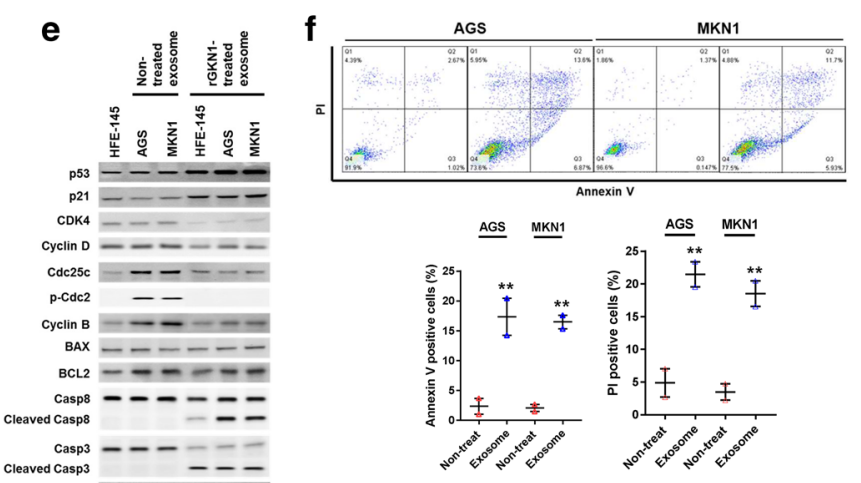

h

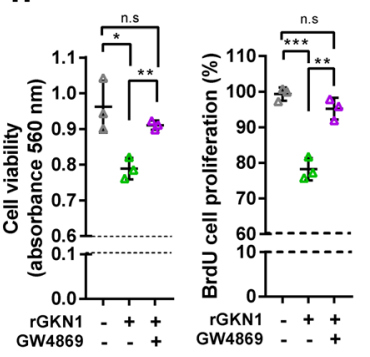

Fig. 2 Exosomes carrying GKN1 protein inhibited cell growth and proliferation. Exosomes derived from AGS and MKN1 cells induced a time-dependent increase in cell viability (a) and proliferation (b) in HFE-145, AGS and MKN1 cells, whereas the exosomes that carry GKN1 decreased cell viability and proliferation $(n=3)$. $\mathbf{c}$ Exosomal GKN1 and rGKN1 markedly decreased cell viability and proliferation in AGS and MKN1 cancer cells over $48 \mathrm{~h}$ after treatment. The inhibitory effect on cell viability of exosomal GKN1 protein was more significant than that of rGKN1 $(n=3)$. d Exosomal GKN1 significantly increased cell populations at the G1 and G2/M phases and decreased cell population at the $\mathrm{S}$ phase $(n=2)$. e Exosomal GKN1 increased the expression of $\mathrm{p} 53$ and $\mathrm{p} 21$ and down-regulated the expression of CDK4, cyclin D, cdc25, p-cdc2, and cyclin B. In addition, exosomal GKN1 induced cleavage of caspase- 3 and -8. f In cell death assay, exosomal GKN1 increased apoptotic cell death in AGS and MKN1 cells $(n=2)$. $\mathbf{g}, \mathbf{h}$ When we treated MKN1 cells with an inhibitor of exosome biogenesis/release (GW4869), exosomal protein concentration significantly decreased $(\mathbf{g} ; n=10)$ and the effects of GKN1 on cell viability and proliferation were markedly inhibited $(\mathbf{h} ; n=3) . P$ values by unpaired two-tailed Student's $t$ test: $* P<0.05, * * P<0.01$, $* * * P<0.001$ and $* * * * P<0.0001$. The center value is the mean, and error bars are sd

AGS and MKN1 cells with PKH26-positive exosomes that carried GKN1 (Fig. 4b), these exosomes were clearly localized in the cytoplasm of AGS and MKN1 cells (Fig. 4c). However, clathrin knockdown dramatically inhibited the internalization of PKH26-positive exosomes into the AGS and MKN1 cells' cytoplasm (Fig. 4c). In these cells, expression of both GKN1 and CD81 was significantly reduced in membrane and not detected in the cytoplasm (Fig. 4d).

\section{Measurement of GKN1 protein levels in sera is useful for gastric cancer diagnosis}

To confirm whether GKN1 protein is present in culture media and human sera, we measured GKN1 concentrations in culture media from HFE-145 cells and human sera by ELISA, after incubation at $70{ }^{\circ} \mathrm{C}$ for $10 \mathrm{~min}$. Under nonheating conditions, we did not detect GKN1 in either total media, exosomes and exosome-depleted media from HFE145 cells (Fig. 5a) or whole serum, exosomes, and exosomefree sera (Fig. 5b). However, the GKN1 concentrations 

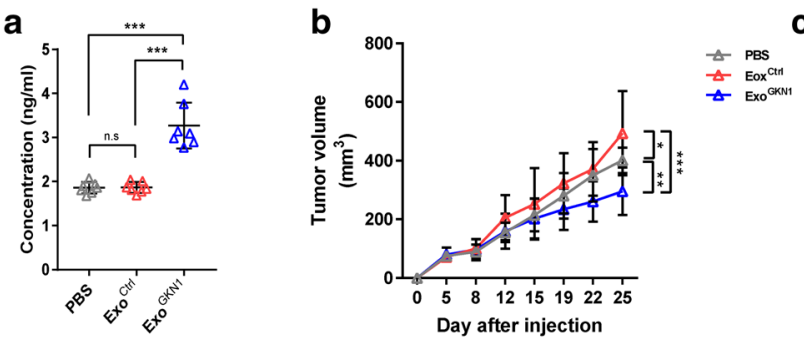

e PBS
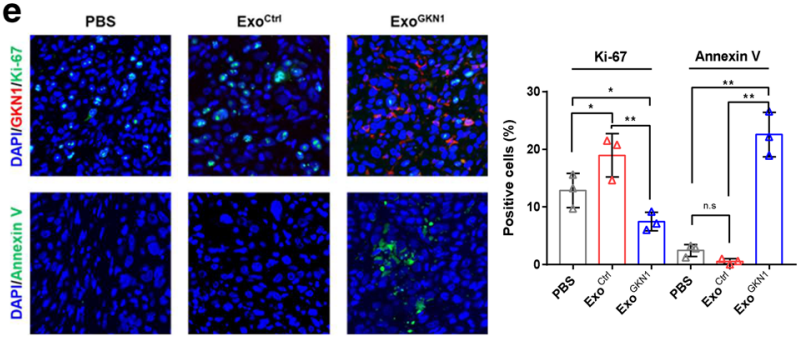

f

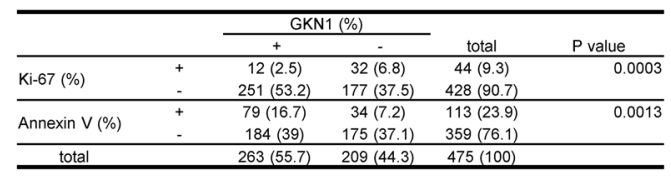

Fig. 3 GKN1-positive exosomes significantly reduced tumor growth in xenograft model. a Serum GKN1 concentration in GKN1 positive exosome-treated group was significantly higher relative to other groups ( $n=7$ mice/group). b-d Mice in the group treated with GKN1-positive exosomes showed relatively reduced tumor volume and weight ( $n=7 \mathrm{mice} / g r o u p)$. e In immunofluorescent analysis, GKN1 expression was detected in the cytoplasm of cancer cells in only GKN1-positive exosome-treated tumor. Growth fraction with Ki-67 staining and apoptosis with Annexin V staining were significantly lower and higher, respectively, in GKN1-positive exosometreated tumors compared with tumors treated with PBS and control exosomes (each group; $n=3$ ). f Percentages of positively stained

in total media, exosomes, and exosome-depleted media treated at $70{ }^{\circ} \mathrm{C}$ for $10 \mathrm{~min}$ were $1.13 \pm 0.06,1.08 \pm 0.1$ and $0.05 \pm 0.04 \mathrm{ng} / \mathrm{ml}$, respectively (Fig. 5a). In ten healthy individuals, the GKN1 concentrations in whole serum and exosomes treated with heat were $6.53 \pm 0.58$ and $6.24 \pm 0.55 \mathrm{ng} / \mathrm{ml}$, respectively (Fig. $5 \mathrm{~b}$ ). In ten gastric cancer patients, they were $3.58 \pm 0.54$ and $3.28 \pm 0.82 \mathrm{ng} / \mathrm{ml}$ in whole serum and exosomes, respectively (Fig. 5b), suggesting that the majority of serum GKN1 is enriched within exosomes. Thus, we analyzed GKN1 concentrations in the whole sera from 100 healthy subjects and 245 patients with gastric, colorectal cancers or hepatocellular carcinomas after heating at $70{ }^{\circ} \mathrm{C}$ for $10 \mathrm{~min}$.

As shown in Fig. 5c, there was a slight association between serum GKN1 levels in healthy individuals and aging, but there was no statistical significance $(P=0.338)$. In addition, there was no difference in serum GKN1 levels between males and females $(P=0.074)$. Interestingly, individuals with atrophic gastritis with and without intestinal
C
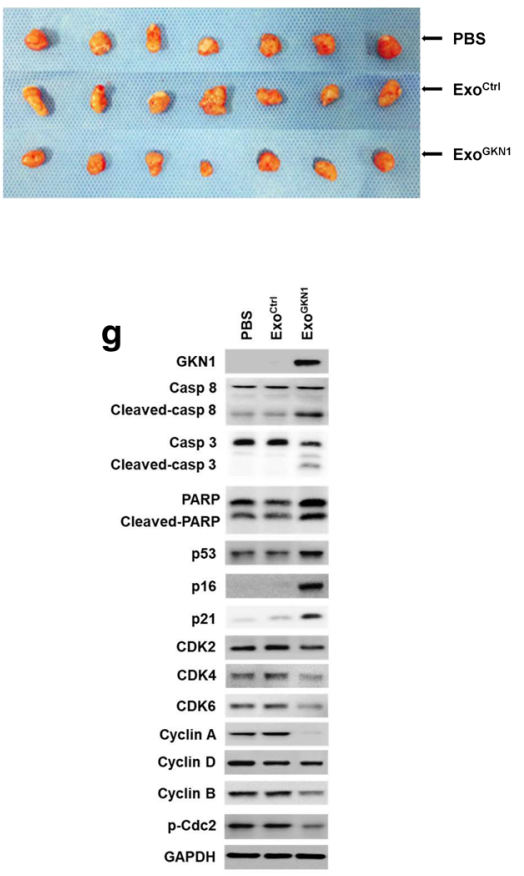

d

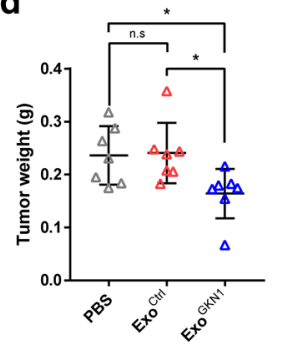

cells for GKN1, Ki-67 and Annexin V in 472 tumor cells treated with GKN1-positive exosomes were 55.7, 9.3, and 23.9\%, respectively. Statistically, expression of GKN1 was inversely correlated with Ki-67 expression and positively correlated with Annexin V staining $(P=0.0003$ and $P=0.0013$, respectively). $\mathbf{g}$ In GKN1-positive exosome-treated tumors, expression of CDK4/6, cyclins A, B and $\mathrm{D}$, and $\mathrm{p}-\mathrm{cdc} 2$, was reduced, while cleaved caspase- $3,-8$, and PARP, p53, p16 and p21 were markedly increased in tumors treated with GKN1-positive exosomes. $P$ values by unpaired two-tailed Student's $t$ test: $* P<0.05$, $* * P<0.01$ and $* * * P<0.001$. The center value is the mean, and error bars are sd

metaplasia (IM) showed serum GKN1 concentrations that were lower than those in subjects without gastritis, but significantly higher than those in patients with gastric cancer (Fig. 5d).

The concentrations of GKN1 in whole sera of 100 healthy subjects and 150 patients with gastric cancer were $6.35 \pm 0.82$ and $3.50 \pm 0.57 \mathrm{ng} / \mathrm{ml}$, respectively (Fig. 5e), indicating that serum GKN1 levels in gastric cancer patients were significantly lower than those in healthy individuals $(P<0.0001)$. Furthermore, GKN1 levels in serum of gastric cancer patients were significantly lower than those of hepatocellular carcinoma $(6.26 \pm 0.95 \mathrm{ng} /$ $\mathrm{ml})$ and colorectal cancer $(6.07 \pm 0.92 \mathrm{ng} / \mathrm{ml})$ (Fig. 5e). Notably, the serum GKN1 concentration discriminated patients with gastric cancer from healthy individuals without atrophic gastritis and subjects with atrophic gastritis without and with IM with the area under the ROC curve (AUC) values of 1.0000, 1.0000, and 0.9964, respectively (Fig. 5f). When we analyzed the ability of 

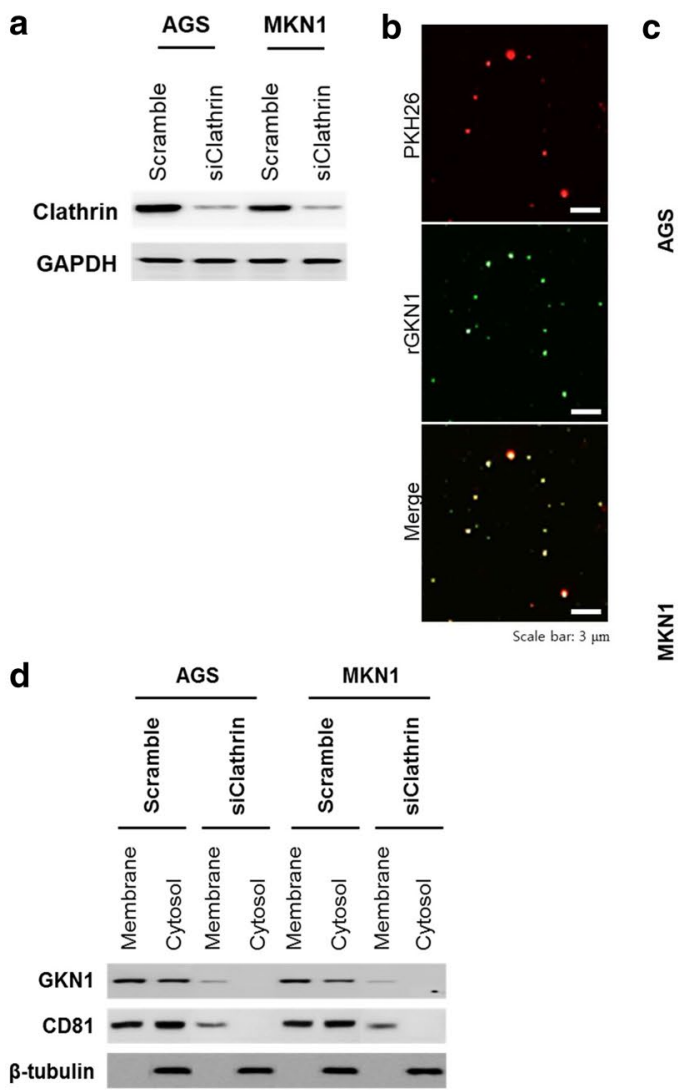
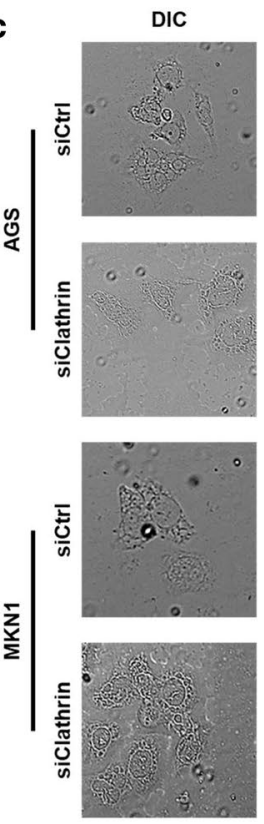
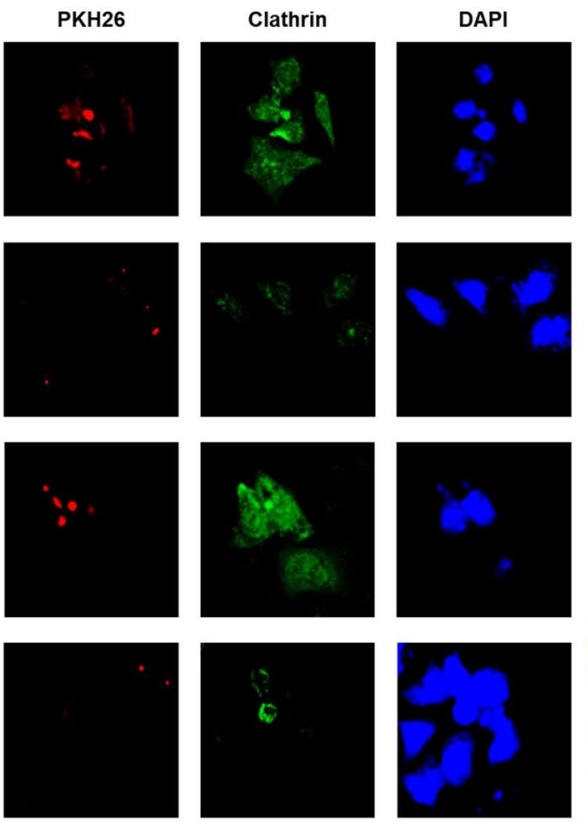
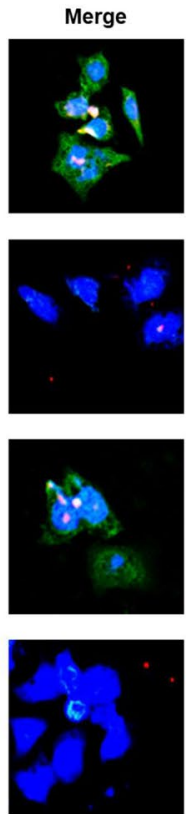

Fig. 4 Clathrin-dependent internalization of GKN1-positive exosomes. a Treatment with siClathrin markedly down-regulated clathrin expression. b We isolated exosomes from AGS gastric cancer cells after treatment with rGKN1. c Localization of exosomes that carry GKN1 protein in AGS and MKN1 gastric cancer cells transfected with siControl and siClathrin. Silencing clathrin dramatically

the serum GKN1 levels to discriminate the patients with gastric cancer from the patients with hepatocellular and colorectal carcinomas, the GKN1 concentration in whole sera predicted the risk of gastric cancer with AUC values of 0.9938 and 0.9987, respectively (Fig. 5g).

Based on the depth of invasion, gastric cancer is classified into early (EGC) and advanced (AGC). Notably, serum GKN1 concentrations in the patients with EGC $(3.75 \pm 0.47 \mathrm{ng} / \mathrm{ml})$ were significantly higher than those in the patients with AGC $(3.38 \pm 0.58 \mathrm{ng} / \mathrm{ml})(P<0.001)$ (Fig. 5h). However, serum GKN1 concentrations in patients with EGC were lower than those tested in subjects with atrophic gastritis and IM $(P<0.001)$ (Fig. 5h). In addition, using GKN1 levels in whole sera, EGC patients can be distinguished from (1) healthy individuals (AUC: 1.00), (2) individuals with atrophic gastritis (AUC: 1.00 ), and (3) individuals with atrophy and IM (AUC: 0.98) (Fig. 5i). inhibited internalization of exosomes that carried GKN1 in these cells. d In western blot assays, GKN1 and CD81 were expressed in the membranes and cytoplasms of AGS and MKN1 gastric cancer cells, whereas we found reduced or lost GKN1 and CD81 expression in clathrin-silenced cells

\section{Exosomes from gastric cancer cells down-regulate NKX6.3 and GKN1 expression in gastric epithelial cells}

Next, we investigated whether exosomes derived from cancer cells down-regulate GKN1 expression in normal gastric epithelial cells. Interestingly, exosomes released from AGS and MKN1 cancer cells markedly inhibited the expression of both GKN1 and NKX6.3, which is a transcriptional factor of GKN1 [24], at mRNA and protein levels and increased expression of c-Myc and Max proteins in HFE-145 cells (Fig. 6a, b). In addition, we observed that the expression levels of c-Myc and Max in exosomes were higher in AGS and MKN1 cancer cells than in HFE-145 cells (Fig. 6c, d). Consistent with this result, ectopic c-Myc expression in HFE-145 cells also increased expression of Max protein and induced loss of GKN1 and NKX6.3 expression (Fig. 6e). 

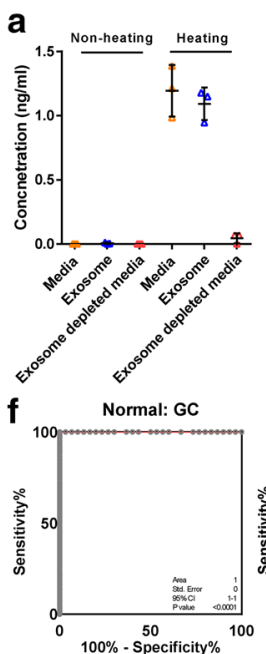

b
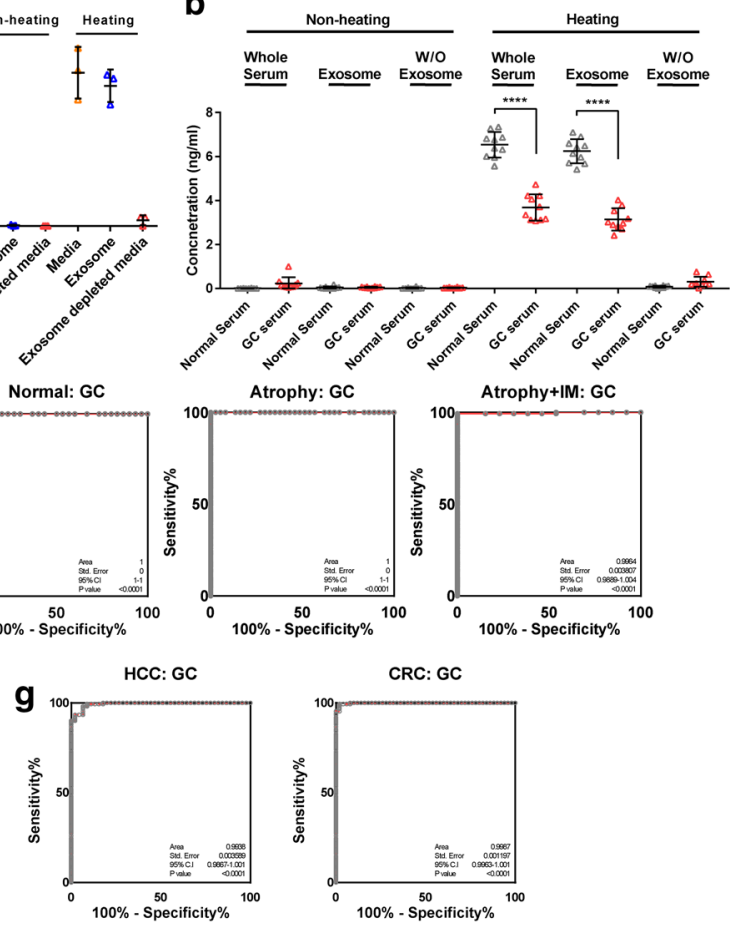

Fig. 5 Diagnostic value of serum GKN1 level for gastric cancer. a In HFE-145 cells, we detected GKN1 protein only in total media and exosomes after heating at $70{ }^{\circ} \mathrm{C}$ for $10 \mathrm{~min}$ but not in the media, exosomes and exosome-depleted media that were not heated (each group; $n=3$ ). b In human sera from both 10 healthy subjects and 10 patients with gastric cancer, we detected GKN1 in whole sera and exosomes heated at $70{ }^{\circ} \mathrm{C}$ for $10 \mathrm{~min}$ but not in the unheated sera. In both healthy subjects and gastric cancer patients, serum GKN1 concentrations in exosomes reached nearly the same levels as those in whole serum (each group; $n=10$ ). $\mathbf{c}$ In 100 healthy subjects, serum GKN1 concentration was not associated with age and gender (duplicated; $n=100$ ). d Serum GKN1 concentrations in individuals with atrophy and IM were significantly lower than those in subjects with normal stomach and atrophic gastritis but higher than those in gastric cancer patients (normal; $n=46$, atrophy; $n=41$, atrophy $+\mathrm{IM} ; n=13$, $G C$ gastric cancer $n=150$, duplicated experiments). e GKN1 levels in the sera of gastric cancer patients were significantly lower than those in the sera of healthy subjects and patients with hepatocellular and colorectal carcinomas (normal; $n=100, G C$ gastric cancer $n=150$,

Next, we attempted to examine whether c-Myc is a transcriptional repressor of NKX6.3. Expectedly, ectopic expression of c-Myc in HFE-145 cells increased the binding activity of c-Myc to the upstream sequences of NKX6.3 and dramatically inhibited NKX6.3 binding to GKN1 promoter region (Fig. 6f). Thus, we conclude that c-Myc in exosomes derived from gastric cancer cells inhibits GKN1 expression in surrounding non-neoplastic gastric epithelial cells, and subsequently induces low serum GKN1 concentration.
C

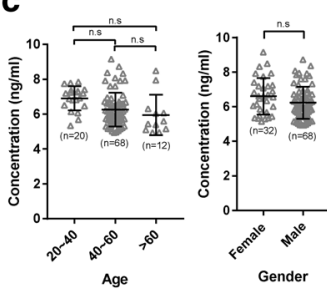

d

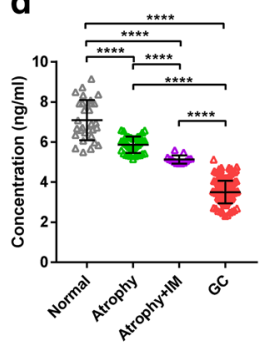

e

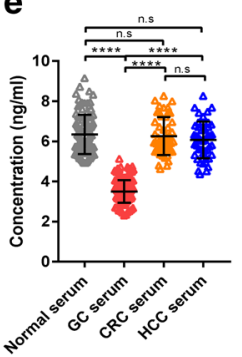

i
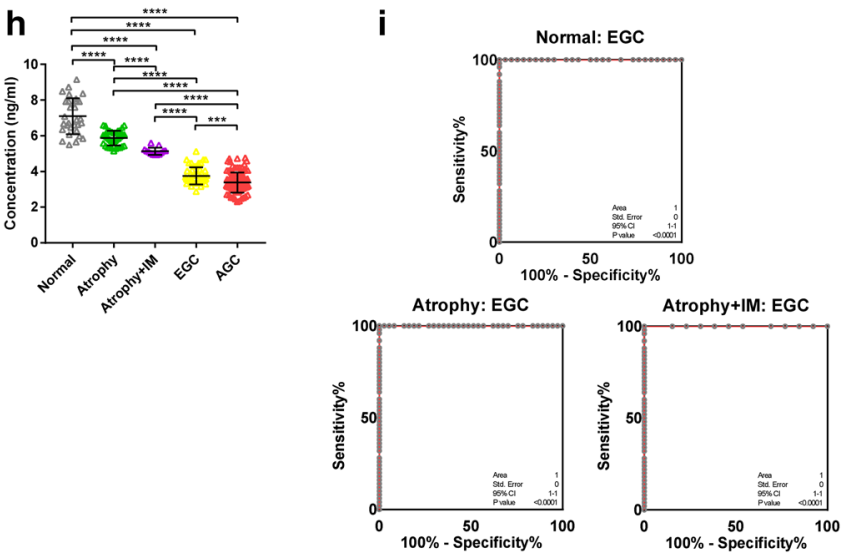

$C R C$ colorectal cancer $n=50, H C C$ hepatocellular carcinoma $n=45$, duplicated experiments). f In ROC curve analysis, serum GKN1 concentrations in gastric cancer patients yielded AUCs of 1.0000, 1.0000 , and 0.9964 (95\% CI $0.9889-1.004 ; P<0.0001$ ) distinguishing healthy individuals with normal stomach and subjects with atrophy without and with IM from gastric cancer patients, respectively. $\mathbf{g}$ Serum GKN1 levels discriminated patients with gastric cancer from patients with hepatocellular and colorectal carcinomas with AUCs of 0.9938 and 0.9987 , respectively. h Serum GKN1 concentrations in patients with EGC were lower than those in subjects with atrophic gastritis with IM and higher than those in patients with AGC (normal; $n=46$, atrophy; $n=41$, atrophy $+\mathrm{IM} ; n=13, E G C$ early gastric cancer $n=50, A G C$ advanced gastric cancer $n=100$, duplicated experiments). i Serum GKN1 concentrations in patients with EGC yielded AUCs of 1.0000, 1.0000 and 0.9892, respectively, distinguishing normal individuals and subjects with atrophic gastritis without and with IM. $P$ values by unpaired two-tailed Student's $t$ test: $* * * P<0.001$ and $* * * * P<0.0001$. The center value is the mean, and error bars are sd

\section{Exosomal GKN1 does not affect cell viability and function of white blood cells}

We further examined the possibility that exosomes that carry GKN1 could modulate cell viability and function of lymphocytes, monocytes, and macrophages. Interestingly, exosomes derived from HFE-145 cells bound to the membrane of THP-1 monocyte and U937 macrophage, but not Jurkat $\mathrm{T}$ cells, in immunofluorescent assay (Fig. 7a). GKN1 was expressed in the membrane fraction of THP-1 monocyte and 

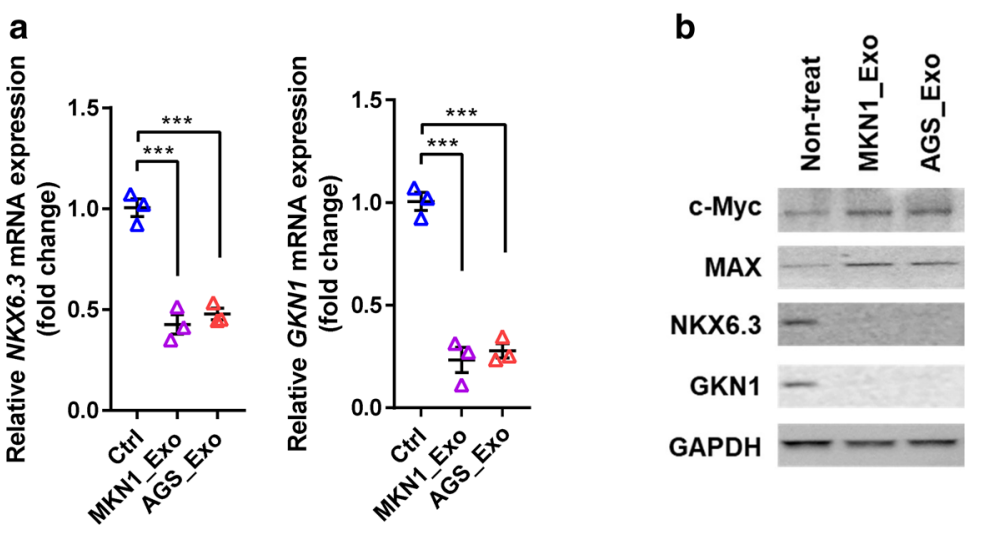

C

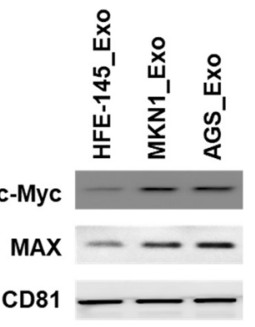

d

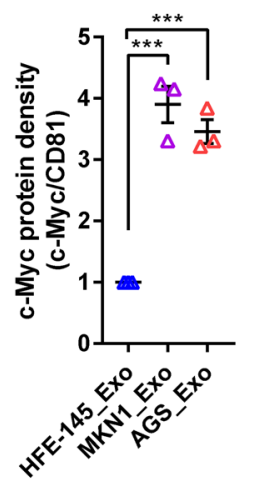

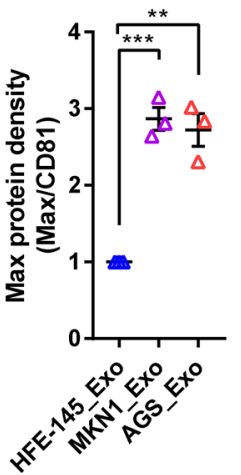

e

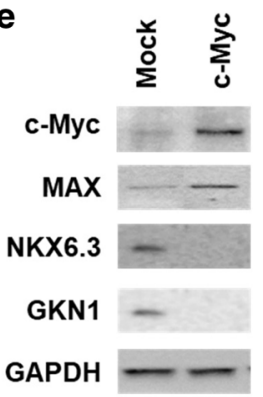

f
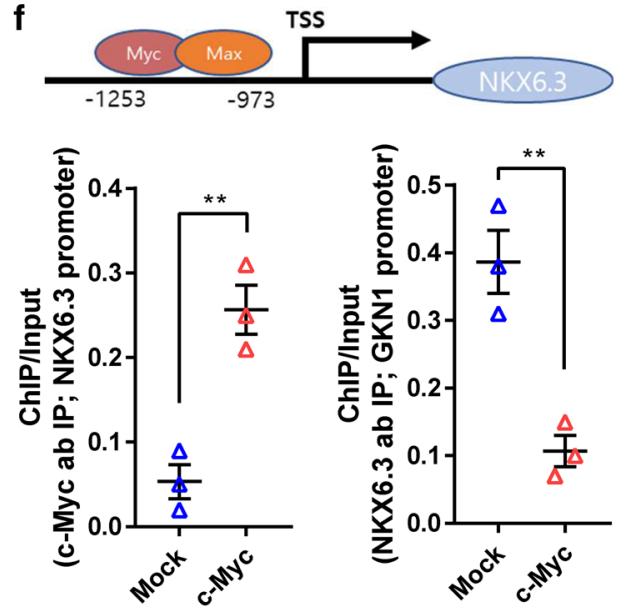

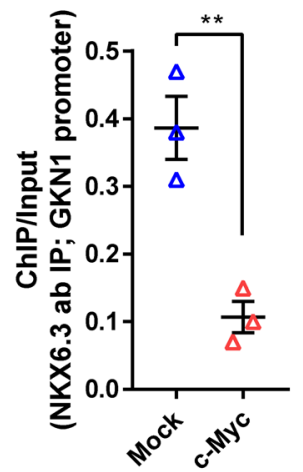

Fig. 6 Exosomal c-Myc protein downregulates NKX6.3 and GKN1 expression. a, b In HFE-145 cells, exosomes derived from MKN1 and AGS cells significantly decreased NKX6.3 and GKN1 expression at mRNA and protein levels (each group; $n=3$ ). c, d Western blot and densitometer analyses showed increased expression of c-Myc and Max in exosomes derived from MKN1 and AGS cells (each group;

U937 macrophage (Fig. 7b). However, the cell viability and expression levels of cytokines were not changed (Fig. 7c, d).

\section{Discussion}

It is well known that GKN1 is packaged within secretary granules and predicted to have a secretory signal peptide [2, $25,26]$. For autocrine and paracrine functions, GKN1 must be internalized by gastric epithelial cells. However, current literature indicates neither GKN1 receptors nor GKN1 secretion and internalization pathways. Here, we aimed to determine whether GKN1 is secreted and internalized in gastric epithelial cells as an exosomal protein.

Here, we found that rGKN1 interacts with 27 exosomal proteins (Fig. 1a). Notably, exosomes from HFE-145 immortalized gastric epithelial cells expressed the GKN1 protein, $n=3)$. e Ectopic c-Myc expression increased Max and completely down-regulated NKX6.3 and GKN1 expression. f In ChIP analysis, ectopic c-Myc expression increased c-Myc binding to NKX6.3 and decreased that of NKX6.3 to GKN1 promoter regions (each group; $n=3) . P$ values by unpaired two-tailed Student's $t$ test: $* * P<0.01$ and $* * * P<0.001$. The center value is the mean, and error bars are sd

but exosomes from AGS and MKN1 gastric cancer cells did not (Fig. 1b). These data are consistent with evidence showing that GKN1 was reduced or lost in gastric cancer [3, 27, 28]. In addition, GKN1 appears to be localized exclusively in exosomes (Fig. 1d, e), suggesting that GKN1 may be secreted into extracellular space as one of exosomal cargo proteins.

Because our previous study showed that rGKN1 significantly inhibits gastric cancer cell viability [5], we hypothesized that biological effects of GKN1 may be induced by exosome-mediated autocrine and paracrine transfer. Previous studies have demonstrated that cancer cell-derived exosomes inhibit tumor cell apoptosis and induce cell proliferation [29, 30]. Consistently, in this study, we found that GKN1-negative exosomes derived from AGS and MKN1 cancer cells increased cell viability and proliferation in HFE145, AGS, and MKN1 cells. In contrast, GKN1-positive 

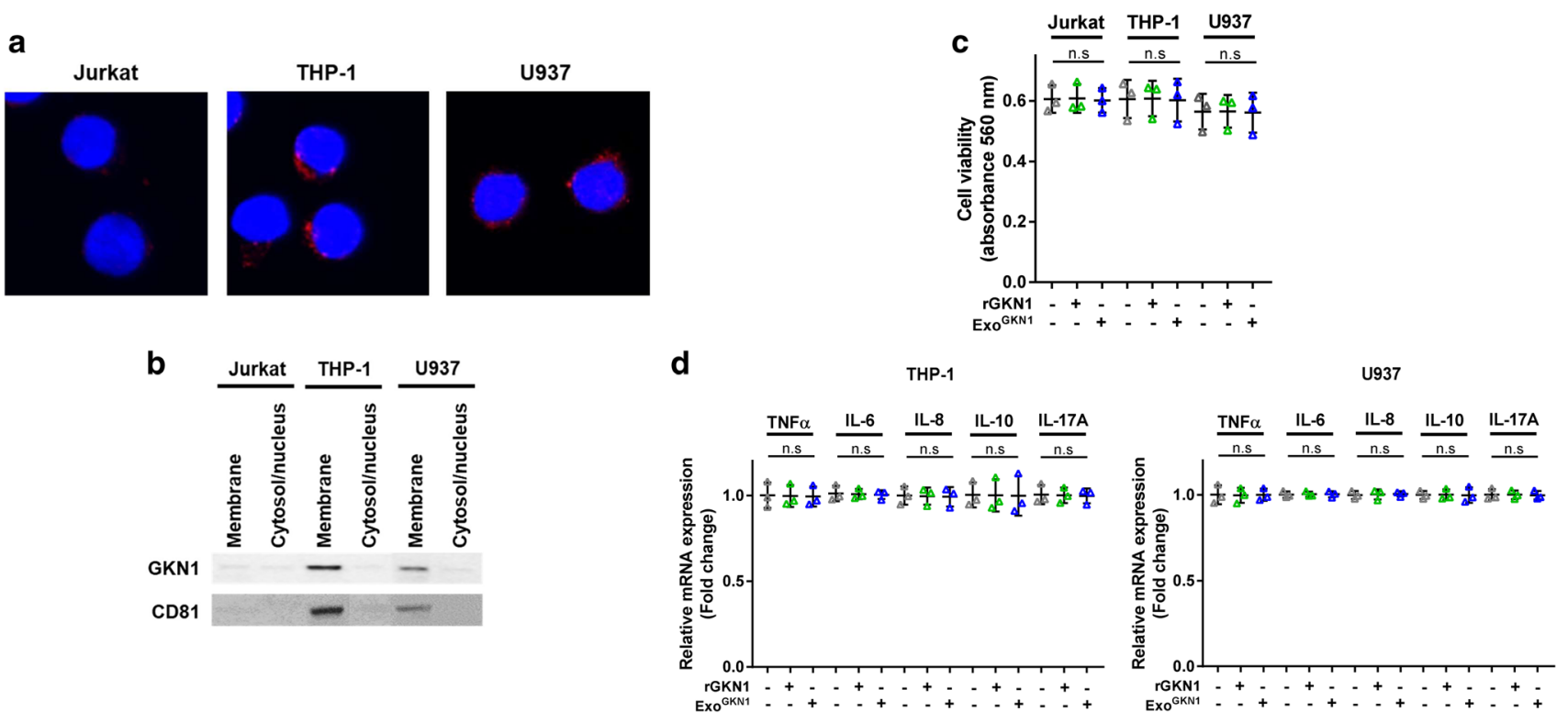

Fig. 7 Exosomal GKN1 did not affect the cell viability of lymphocytes and macrophages. a GKN1-positive exosomes derived from HFE-145 cells were attached to plasma membranes of THP-1 monocytes and U937 macrophages, but not Jurkat T cells. b In western blot analysis, we detected GKN1 protein only in the membrane fractions of these cells. c, d Exosomes that carry GKN1 did not affect cell viability and cytokine expression of THP-1 monocytes and U937 macrophages $(n=3)$ exosomes markedly inhibited cell viability and cell proliferation (Fig. 2a, b) and the effects of exosomal GKN1 on cell proliferation and apoptosis were stronger than those of rGKN1 (Fig. 2c). Consistent with the previous results [5, 28, 31], exosomal GKN1 induced G1 and G2/M arrests and apoptosis by regulating expression of proteins related to cell cycle and apoptosis (Fig. 2d-f and supplementary Fig. 2). In addition, GW4869, an inhibitor of exosome biogenesis/ release, dramatically suppressed the effects of GKN1 on cell growth (Fig. 2h). In the nude mouse xenograft model, exosomal GKN1 significantly suppressed the growth of subcutaneous transplanted tumors of MKN1 cells by inhibiting cell proliferation and inducing apoptosis (Fig. 3). These findings strongly suggest that secreted exosomal GKN1 may be internalized into gastric epithelial cells and thereby alter a set of signaling pathways involved in regulation of cell growth and apoptosis. Because exosomes have limitless therapeutic potential for the treatment of cancer [32], it is plausible that modulating exosomes that carry GKN1 and stimulating its anti-cancerous effects could significantly impact the development of novel gastric cancer treatments, which will ultimately achieve the goal of gastric cancer prevention and remission.

For these phenotypic effects to occur, exosomes that carry GKN1 need to be fused with gastric epithelial cell membranes and be taken up into cells. EVs are usually taken up into endosomal compartments via endocytosis [18-20], and EV uptake is most likely dependent on the signaling status of recipient cells and the protein complement of the vesicle [33]. Because the internalization of various cargo proteins and lipids from the mammalian cell surface occurs through clathrin and lipid-raft endocytic pathway [32], we treated AGS and MKN1 cells with rGKN1 after silencing clathrin with siClathrin. Interestingly, internalization of exosomes that carry GKN1 was dramatically inhibited in clathrin-silenced AGS and MKN1 cells (Fig. 4b, c), suggesting that clathrin-mediated endocytosis may be the main pathway for internalization of GKN1-positive exosomes into gastric epithelial cells. Although the mechanism that underlies fusion and uptake of these exosomes to the gastric epithelium remains to be clearly identified, we here provide direct evidence that GKN1 can induce autocrine and paracrine signaling through exosome exchange in gastric epithelial cells. Additional characterization of the pathways of internalization of GKN1-positive exosomes will elucidate the mechanisms of exosome-mediated therapeutic targets.

Recently, Villano et al. reported that GKN1 mRNA in sera is not an informative biomarker for this cancer [34]. Here, to investigate whether GKN1 protein is present in sera as an exosomal protein, we analyzed GKN1 concentrations in 10 sera with and without heating at $70{ }^{\circ} \mathrm{C}$ for $10 \mathrm{~min}$. Expectedly, we detected GKN1 in whole sera and serum exosomes, and its levels in exosomes reached nearly the similar to those of whole serum in both healthy subjects and gastric cancer patients (Fig. 5a, b). Thus, we conclude that vast majority of serum GKN1 is present within exosomes and that the protein could be delivered to non-gastric tissues as circulating exosomes. 
Next, to evaluate the diagnostic value of serum GKN1, we examined GKN1 concentrations after heating at $70{ }^{\circ} \mathrm{C}$ for $10 \mathrm{~min}$, in whole sera from 100 healthy subjects and 245 patients with gastric, colorectal, and hepatocellular carcinoma. Interestingly, GKN1 levels in the sera of gastric cancer patients were significantly lower than those in the sera of healthy subjects and the patients with hepatocellular and colorectal carcinomas $(P<0.0001$; Fig. 5e). Notably, the serum GKN1 levels discriminated the patients with gastric cancer from healthy subjects and patients with hepatocellular and colorectal carcinomas (Fig. 5g). In addition, GKN1 concentrations in whole sera discriminated the patients with EGC from healthy individuals without atrophic gastritis and subjects with atrophic gastritis without and with IM (Fig. 5i). EVs protect their cargo from the attack of nuclease and proteases [35]. EVs are well suited for biomarker analyses that may increase sensitivity and/or specificity $[35,36]$. Thus, we conclude that GKN1 protein in serum may be an informative diagnostic biomarker for gastric cancer. Future studies with large populations are strongly needed to verify these initial observations.

These observations that serum GKN1 concentrations were lower in patients with gastric cancer prompted us to examine if cancer-derived exosomes inhibit the GKN1 expression in surrounding non-neoplastic gastric epithelial cells. In HFE-145 cells, exosomes released from AGS and MKN1 cancer cells had the potential to inhibit both GKN1 and NKX6.3 expression at mRNA and protein levels (Fig. 6a, b) by overexpression of c-Myc and Max (Fig. 6b). Consistent with the report that cancer exosomes alter the transcriptomes of target cells and stimulate non-tumorigenic epithelial cells to form tumors [37], it is most likely that exosomes derived from gastric cancer cells inactivate gastric tumor suppressors NKX6.3 and GKN1 in surrounding nonneoplastic gastric epithelial cells and subsequently decrease serum GKN1 concentrations. Additional studies are required to verify that cancer-derived exosomes instigate the normal cells to participate in progression of gastric cancer.

Because we detected GKN1 in human sera, we examined whether exosomes carrying GKN1 have an influence on white blood cells. GKN1-positive exosomes derived from HFE-145 cells were detected onto plasma membranes of THP-1 monocytes and U937 macrophages (Fig. 7a, b). However, no significant effect of exosomal GKN1 on cell viability and cytokine expression was seen in these cells (Fig. 7c, d). Thus, we can speculate that exosomal GKN1 may be used as a therapeutic target for gastric cancer without hematologic side effects.

In summary, an important observation of this study is that GKN1 was secreted to extracellular space as an exosomal cargo protein and was internalized through clathrinmediated endocytosis. Exosomal GKN1 inhibited cell proliferation and induced apoptosis in gastric cancer cells and xenograft tumors. In addition, GKN1 concentrations in the sera of patients with gastric cancer were significantly lower than those in healthy subjects and patients with hepatocellular and colorectal carcinomas $(P<0.0001)$, suggesting that serum GKN1 may be an informative biomarker for the diagnosis of gastric cancer. Finally, we provided the first evidence that human gastric epithelial cells naturally secrete and internalize GKN1 as an exosomal protein and that GKN1 may be a potential diagnostic and therapeutic target for achieving gastric cancer remission.

Acknowledgements This study was supported by the Basic Science Research Program through the National Research Foundation of Korea (NRF) funded by the Ministry of Education, Science and Technology (2015R1A2A2A01002531 to W.S.P) and a grant of the Korea Health Technology R\&D Project through the Korea Health Industry Development Institute (KHIDI), funded by the Ministry of Health \& Welfare, Republic of Korea (HI17C0364 to H.H). We thank Dr. SeongYeob Ryu, Department of Gastroenterologic Surgery, Chonnam National University Hwasun Hospital, 160, Ilsim-ri, Hwasun-eup, Hwasungun, Jeollanam-do, 519-809, Korea, for providing the gastric cancer samples with clinical information.

Author contributions JHY and IHH performed the majority of experiments, analyzed the data, interpreted the data, funding acquisition and contributed to writing the manuscript. OK writing review and edited the manuscript. AH and STD provided the HFE-145 cells before publication. SWN and JYL writing review and edited the manuscript and helped in the interpreted the data. HH and WSP conceived the project, interpreted the data, funding acquisition and wrote the manuscript.

\section{Compliance with ethical standards}

Conflict of interest The authors have declared no conflicts of interest. Dr. Park reports grants from The Basic Science Research Program through the National Research Foundation of Korea (NRF) funded by the Ministry of Education, Science and Technology (2015R1A2A2A01002531 to W.S.P), grants from The Korea Health Technology R\&D Project through the Korea Health Industry Development Institute (KHIDI), funded by the Ministry of Health \& Welfare, Republic of Korea (HI17C0364 to H.H), during the conduct of the study; In addition, Dr. Park has a patent Diagnosis of gastric cancer using gastrokine 1 protein within blood pending, and a patent Anticancer composition including exosomal GKN1 protein pending.

Ethical standards All procedures followed were in accordance with the ethical standards of the responsible committee on human experimentation (institutional and national) and with the Helsinki Declaration of 1964 and later versions. Informed consent to be included in the study, or the equivalent, was obtained from all patients. For studies with animals, all institutional and national guidelines for the care and use of laboratory animals were followed.

Open Access This article is distributed under the terms of the Creative Commons Attribution 4.0 International License (http://creativeco mmons.org/licenses/by/4.0/), which permits unrestricted use, distribution, and reproduction in any medium, provided you give appropriate credit to the original author(s) and the source, provide a link to the Creative Commons license, and indicate if changes were made. 


\section{References}

1. Yoshikawa Y, Mukai H, Hino F, Asada K, Kato I. Isolation of two novel genes, down-regulated in gastric cancer. Jpn J Cancer Res. 2000;91:459-63.

2. Martin TE, Powell CT, Wang Z, Bhattacharyya S, Walsh-Reitz $\mathrm{MM}$, Agarwal K, et al. A novel mitogenic protein that is highly expressed in cells of the gastric antrum mucosa. Am J Physiol Gastrointest Liver Physiol. 2003;285:G332-43.

3. Oien KA, McGregor F, Butler S, Ferrier RK, Downie I, Bryce $\mathrm{S}$, et al. Gastrokine 1 is abundantly and specifically expressed in superficial gastric epithelium, down-regulated in gastric carcinoma, and shows high evolutionary conservation. J Pathol. 2004;203:789-97.

4. Walsh-Reitz MM, Huang EF, Musch MW, Chang EB, Martin TE, Kartha S, et al. AMP-18 protects barrier function of colonic epithelial cells: role of tight junction proteins. Am J Physiol Gastrointest Liver Physiol. 2005;289:G163-71.

5. Yoon JH, Choi YJ, Choi WS, Ashktorab H, Smoot DT, Nam SW, et al. GKN1-miR-185-DNMT1 axis suppresses gastric carcinogenesis through regulation of epigenetic alteration and cell cycle. Clin Cancer Res. 2013;19:4599-610.

6. Kim O, Yoon JH, Choi WS, Ashktorab H, Smoot DT, Nam SW, et al. Gastrokine 1 inhibits gastrin-induced cell proliferation. Gastric Cancer. 2016;19:381-91.

7. Yoon JH, Choi WS, Kim O, Park WS. The role of gastrokine 1 in gastric cancer. J Gastric Cancer. 2014;14:147-55.

8. Chen P, Li YC, Toback FG. AMP-18 targets p21 to maintain epithelial homeostasis. PLoS One. 2015;10:e0125490.

9. Jung T, Castellana D, Klingbeil P, Cuesta Hernández I, Vitacolonna M, Orlicky DJ, et al. CD44v6 dependence of premetastatic niche preparation by exosomes. Neoplasia. 2009;11:1093-105.

10. Qu JL, Qu XJ, Zhao MF, Teng YE, Zhang Y, Hou KZ, et al. Gastric cancer exosomes promote tumour cell proliferation through PI3K/Akt and MAPK/ERK activation. Dig. Liver Dis. 2009;41:875-80

11. Raposo G, Nijman HW, Stoorvogel W, Liejendekker R, Harding $\mathrm{CV}$, Melief CJ, et al. B lymphocytes secrete antigen-presenting vesicles. J Exp Med. 1996;183:1161-72.

12. Mulcahy LA, Pink RC, Carter DR. Routes and mechanisms of extracellular vesicle uptake. J Extracell Vesicles. 2014;3:24641-54

13. Raposo G, Stoorvogel W. Extracellular vesicles: exosomes, microvesicles, and friends. J Cell Biol. 2013;200:373-83.

14. Valadi H, Ekström K, Bossios A, Sjöstrand M, Lee JJ, Lötvall JO. Exosome-mediated transfer of mRNAs and microRNAs is a novel mechanism of genetic exchange between cells. Nat Cell Biol. 2007;9:654-9.

15. Huang SH, Li Y, Zhang J, Rong J, Ye S. Epidermal growth factor receptor-containing exosomes induce tumor-specific regulatory $\mathrm{T}$ cells. Cancer Investig. 2013;31:330-5.

16. Rana S, Zöller M. Exosome target cell selection and the importance of exosomal tetraspanins: a hypothesis. Biochem Soc Trans. 2011;39:559-62.

17. Record M, Carayon K, Poirot M, Silvente-Poirot S. Exosomes as new vesicular lipid transporters involved in cell-cell communication and various pathophysiologies. Biochim Biophys Acta. 2014;1841:108-120.

18. Montecalvo A, Larregina AT, Shufesky WJ, Stolz DB, Sullivan ML, Karlsson JM, et al. Mechanism of transfer of functional microRNAs between mouse dendritic cells via exosomes. Blood. 2012;119:756-66.
19. Morelli AE, Larregina AT, Shufesky WJ, Sullivan ML, Stolz DB, Papworth GD, et al. Endocytosis, intracellular sorting, and processing of exosomes by dendritic cells. Blood. 2004;104:3257-66.

20. Escrevente C, Keller S, Altevogt P, Costa J. Interaction and uptake of exosomes by ovarian cancer cells. BMC Cancer. 2011;11:108.

21. Guang W, Ding H, Czinn SJ, Kim KC, Blanchard TG, Lillehoj EP. Muc1 cell surface mucin attenuates epithelial inflammation in response to a common mucosal pathogen. J Biol Chem. 2010;285:20547-57.

22. Yoon JH, Eun JW, Choi WS, Kim O, Nam SW, Lee JY, et al. NKX6.3 is a transcription factor for Wnt/ $\beta$-catenin and RhoGTPase signaling-related genes to suppress gastric cancer progression. EBioMedicine. 2016; 9: 97-109.

23. Klibi J, Niki T, Riedel A, Pioche-Durieu C, Souquere S, Rubinstein E, et al. Blood diffusion and Th1-suppressive effects of galectin-9-containing exosomes released by EpsteinBarr virus-infected nasopharyngeal carcinoma cells. Blood. 2009; 113:1957-66.

24 Yoon JH, Choi WS, Kim O, Choi SS, Lee EK, Nam SW, et al. NKX6.3 controls gastric differentiation tumorigenesis. Oncotarget. 2015;6:28425-39.

25 Toback FG, Walsh-Reitz MM, Musch MW, Chang EB, Del Valle J, Ren H, et al. Peptide fragments of AMP-18, a novel secreted gastric antrum mucosal protein, are mitogenic and motogenic. Am J Physiol Gastrointest Liver Physiol. 2003;285:G344-53.

26 Xing R, Li W, Cui J, Zhang J, Kang B, Wang Y, et al. Gastrokine 1 induces senescence through $\mathrm{p} 16 / \mathrm{Rb}$ pathway activation in gastric cancer cells. Gut. 2012;61:43-52.

27 Oien KA, Vass JK, Downie I, Fullarton G, Keith WN. Profiling, comparison and validation of gene expression in gastric carcinoma and normal stomach. Oncogene. 2003;22:4287-300.

28 Yoon JH, Song JH, Zhang C, Jin M, Kang YH, Nam SW, et al. Inactivation of the Gastrokine 1 gene in gastric adenomas and carcinomas. J Pathol. 2011;223:618-25.

29 Valenti R, Huber V, Iero M, Filipazzi P, Parmiani G, Rivoltini L. Tumor-released microvesicles as vehicles of immunosuppression. Cancer Res. 2007;67:2912-5.

30 Yang L, Wu XH, Wang D, Luo CL, Chen LX. Bladder cancer cell-derived exosomes inhibit tumor cell apoptosis and induce cell proliferation in vitro. Mol Med Rep. 2013;8:1272-8.

31 Yoon JH, Cho ML, Choi YJ, Back JY, Park MK, Lee SW, et al. Gastrokine 1 regulates NF- $\mathrm{KB}$ signaling pathway and cytokine expression in gastric cancers. J Cell Biochem. 2013;114:1800-9.

32 Le Roy C, Wrana JL. Clathrin- and non-clathrin-mediated endocytic regulation of cell signalling. Nat Rev Mol Cell Biol. 2005;6:112-26.

33 Mulcahy LA, Pink RC, Carter DR. Routes and mechanisms of extracellular vesicle uptake. J Extracell Vesicles. 2014;3:24641.

34 Villano V, Di Stadio CS, Federico A, Altieri F, Miselli G, De Palma M, et al. Gastrokine 1 mRNA in human sera is not informative biomarker for gastric cancer. J Negat Results Biomed. 2016;15:14

35 Taylor DD, Gercel-Taylor C. MicroRNA signatures of tumorderived exosomes as diagnostic biomarkers of ovarian cancer. Gynecol Oncol. 2008;110:13-21.

36 Dijkstra S, Birker IL, Smit FP, Leyten GH, de Reijke TM, van Oort IM, et al. Prostate cancer biomarker profiles in urinary sediments and exosomes. J Urol. 2014;191:1132-8.

37 Melo SA, Sugimoto H, O'Connell JT, Kato N, Villanueva A, Vidal A, et al. Cancer exosomes perform cell-independent microRNA biogenesis and promote tumorigenesis. Cancer Cell. 2014;26:707-21. 